of $z$ and each $S_{j}$ is even. Let $s$ be the number of points of the orbit and $q$ any index in $Q$. For $r<s,(h z)^{r}(p, q)$ differs from $(p, q)$ in the first coordinate; but $(h z)^{8}(p, q)=(p, q)$. Thus every element of $G$ has an odd cycle. As we noted above, this implies [1] the existence of a fair game of $2^{k}\left(2^{l}-1\right)$ players.

\title{
REFERENCES
}

1. J. R. Isbell, Homogeneous games, Math. Student vol. 25 (1957) pp. 123-128.

2. R. D. Luce and H. Raiffa, Games and decisions: Introduction and critical survey, New York, 1957.

3. J. Singer, $A$ theorem in finite projective geometry and some applications to number theory, Trans. Amer. Math. Soc. vol. 43 (1938) pp. 377-385.

UNIVERSITY OF WASHINGTON

\section{ON INDUCED TOPOLOGIES IN QUASI-REFLEXIVE BANACH SPACES ${ }^{1}$}

\section{LARRY C. HUNTER}

1. Introduction. Let $\pi$ denote the canonical isomorphism of a Banach space $X$ into its second conjugate space $X^{* *}$. An example is given by James [4] of a space $X$ for which $X$ is separable, $X$ is not reflexive, $X$ is isomorphic to $X^{* *}$, and $X^{* *} / \pi(X)$ is one-dimensional. Civin and Yood undertook a more complete investigation of Banach spaces $X$ such that $X^{* *} / \pi(X)$ is (finite) $n$-dimensional and called such spaces quasi-reflexive Banach spaces of order $n$. If $Q$ is a subset of $X^{*}$, let $\sigma(X, Q)$ denote the least fine topology for $X$ such that all $x^{*} \in Q$ are continuous. In [1] Civin and Yood establish the following result.

THEOREM A. The following statements are equivalent:

(1) $X$ is quasi-reflexive of order $n$.

(2) There is an equivalent norm for $X$ such that $X^{*}=Q \oplus R$ where $Q$ is a total closed linear manifold such that the unit ball of $X$ is compact in $\sigma(X, Q)$ and $R$ is an n-dimensional linear manifold.

It is the purpose of this paper to study properties of the topologies $\sigma(X, Q)$, where $X^{*}=Q \oplus R, Q$ is a total closed linear manifold, and

Received by the editors December 26, 1958 and, in revised form, May 18, 1959.

1 This paper contains part of a doctoral dissertation written at the University of Oregon under the direction of Paul Civin and was supported in part by the National Science Foundation research grant NSF-G2573. 
$R$ is $n$-dimensional. It is shown that $\sigma(X, Q)$ is nothing more than the $w^{*}$-topology on $X$ when $X$ is considered as the conjugate space of $Q$.

2. Notation. Let $X$ be a Banach space. Let $\pi$ be the canonical isomorphism of $X$ into $X^{* *}$, its second conjugate space. For a subset $A$ of $X, A^{+}$will designate the annihilator of $A$ in $X^{*}$, and $A^{++}$the annihilator of $A^{+}$in $X^{* *}$. For a set $B$ in $X^{*}, B^{-}$will denote the annihilator of $B$ in $X$. When we write $X=C \oplus D$, we shall mean that $C$ and $D$ are closed linear manifolds of $X$, that $X$ is the linear span of $C$ and $D$, and $C \cap D=0$. We define $S_{r}=\{x \in X:\|x\| \leqq r\}$.

3. Preliminary results. If $X$ is a quasi-reflexive Banach space of order $n$, then $X^{* *}=\pi(X) \oplus L$ where $L$ is an $n$-dimensional linear manifold. Civin and Yood note that $X^{*}=Q \oplus R$ where $Q=L^{-}$is total and $R$ is $n$-dimensional. In the proof of Theorem $\mathrm{A}$, they show that for $Q=L^{-}$there is an equivalent norm for $X$ such that the unit ball of $X$ is compact in $\sigma(X, Q)$.

The following question can then be posed. If $X$ is a quasi-reflexive space of order $n$ and $X^{*}=Q_{0} \oplus R_{0}$ where $Q_{0}$ is total and $R_{0}$ is $n$ dimensional, is there an equivalent norm for $X$ in which the unit ball is compact in $\sigma\left(X, Q_{0}\right)$ ? The following theorem shows that all decompositions of $X^{*}$ of the above type arise from considering the annihilators of the $n$-dimensional pieces of the second conjugate space of $X$.

3.1. Theorem. If $X$ is a quasi-reflexive Banach space of order $n$ and if $X^{*}=Q_{0} \oplus S_{0}$ where $Q_{0}$ is total and $S_{0}$ is n-dimensional, then:

(i) $X^{* *}=\pi(X) \oplus Q_{0}^{+}$,

(ii) there is an equivalent norm for $X$ such that the unit ball of $X$ is compact in $\sigma\left(X, Q_{0}\right)$,

(iii) $\|x\|=\sup _{x^{*} \in Q_{0} ;\left\|x^{*}\right\|=1}\left|x^{*}(x)\right|$ if $X$ has the norm for which the unit ball is compact in $\sigma(X, Q)$.

Proof. (i) Suppose that $x^{* *} \in \pi(X) \cap Q_{0}^{+}$. Then $x^{* *}=\pi(x)$ for some $x \in X$ and for all $y^{*} \in Q_{0}, x^{* *}\left(y^{*}\right)=y^{*}(x)=0$. Since $Q_{0}$ is total, $x=0$, and hence $\pi(X) \cap Q_{0}^{+}=0$. Since $X^{* *} / \pi(X)$ has dimension $n$, it follows that $Q_{0}^{+}$has dimension $r \leqq n$. Let $x_{1}^{* *}, x_{2}^{* *}, \cdots, x_{r}^{* *}$ be a basis for $Q_{0}^{+}$and select $x_{1}^{*}, x_{2}^{*}, \cdots, x_{r}^{*} \in X^{*}$ such that $x_{i}^{* *}\left(x_{j}^{*}\right)=\delta_{i j}, i, j=1$, $2, \cdots, r$. Let $R$ be the subspace of $X^{*}$ generated by $x_{1}^{*}, \cdots, x_{r}^{*}$. It is easily seen that $X^{*}=Q_{0} \oplus R$ and thus $X^{*} / Q_{0}$ has dimension $r$. But $X^{*}=Q_{0} \oplus S_{0}$ where $S_{0}$ is $n$-dimensional, so $X^{*} / Q_{0}$ has dimension $n$. Hence $r=n$. 
(ii) Since $X^{* *}=\pi(X) \oplus Q_{0}^{+}$, the result follows immediately from the proof of Theorem A.

(iii) This follows immediately from Theorem 7 of [3].

In view of 3.1, we adopt the following convention. When we say that $X$ is a quasi-reflexive Banach space, $X^{*}=Q \oplus R$ where $Q$ is total and $R$ is $n$ dimensional, we shall always mean that $X$ is to be considered in its equivalent norm so that its unit ball is compact in $\sigma(X, Q)$.

4. Induced topologies. In this section the topologies induced on $X$ by the decompositions $X^{*}=Q \oplus R, Q$ total, $R n$-dimensional, are characterized as $w^{*}$-topologies.

4.1. Theorem. If $X$ is a quasi-reflexive Banach space, $X^{*}=Q \oplus R$ where $Q$ is total and $R$ is $n$-dimensional, then $X$ is equivalent to $Q^{*}$ under the mapping $\nu: X \rightarrow Q^{*}$ defined by $\nu(x)\left(x^{*}\right)=x^{*}(x)$, all $x^{*} \in Q$.

Proof. $\nu(x)$ is the contraction of $\pi(x)$ to $Q$. This is linear and 1-1, since $Q$ is total. By Theorem 9 of [2], $\pi(x)$ and its contraction to $Q$ have the same norm.

Hence $\sigma(X, Q)$ is merely the $w^{*}$-topology on $X$ when $X$ is considered as the conjugate space of $Q$ and properties which hold for general conjugate spaces thus hold for quasi-reflexive Banach spaces.

\section{BIBLIOGRAPHY}

1. Paul Civin and Bertram Yood, Quasi-reflexive spaces, Proc. Amer. Math. Soc. vol. 8 (1957) pp. 906-911.

2. J. Dieudonné, La dualité dans les espaces vectoriels topologiques, Ann. Sci. Ecole Norm. Sup. vol. 59 (1942) pp. 107-139.

3. J. Dixmier, Sur un theorème de Banach, Duke Math. J. vol. 15 (1948) pp. 10571071.

4. Robert C. James, Bases and reflexivity of Banach spaces, Ann. of Math. vol. 52 (1950) pp. 518-527.

Sylvania Electric Products, Inc., Mt. View, California 